\title{
Asymptotic results for the absorption time of telegraph processes with elastic boundary at the origin*
}

\author{
Claudio Macci ${ }^{\dagger} \quad$ Barbara Martinucci ${ }^{\ddagger} \quad$ Enrica Pirozzi ${ }^{\S}$
}

\begin{abstract}
We consider a telegraph process with elastic boundary at the origin studied recently in the literature (see e.g. [5]). It is a particular random motion with finite velocity which starts at $x \geq 0$, and its dynamics is determined by upward and downward switching rates $\lambda$ and $\mu$, with $\lambda>\mu$, and an absorption probability (at the origin) $\alpha \in(0,1]$. Our aim is to study the asymptotic behavior of the absorption time at the origin with respect to two different scalings: $x \rightarrow \infty$ in the first case; $\mu \rightarrow \infty$, with $\lambda=\beta \mu$ for some $\beta>1$ and $x>0$, in the second case. We prove several large and moderate deviation results. We also present numerical estimates of $\beta$ based on an asymptotic Normality result for the case of the second scaling.
\end{abstract}

Keywords: finite velocity, random motion, large deviations, moderate deviations.

Mathematical Subject Classification: 60F10, 60J25, 60K15.

\section{Introduction}

The (integrated) telegraph process is an alternating random motion with finite velocity, and has several applications in different fields (for instance in physics, finance and mathematical biology). The literature on the telegraph process and its generalizations is quite large. In this paper we refer to a recent model with elastic barrier studied in [5], where it is possible find several references. Here we only recall some of them.

We start with some references that studied the solution of the telegraph equation; see e.g. 12 and [15]. Among the first references that studied some probabilistic aspects, we recall [18] and [9]. Moreover we also cite [19] and 20. where the telegraph process in the presence of reflecting and absorbing barriers was investigated. Among the more recent references with some generalizations, we recall 22 for a model with random velocities, 33 for a damped telegraph process, 1] for a model driven by certain random trials, [4] for a telegraph process perturbed by a Brownian motion, 6] and [10] for certain multivariate extensions, and [21] for a model with jumps having some interest in finance. Finally, since in this paper we prove results on large deviations, we also recall [17] (see also some references cited therein) and the previous paper [16].

We also cite some references on stochastic processes with elastic barriers: [7] and [8] for the Wiener process, [11] for some diffusion processes and, more recently, [13 and [14] for the Langevin process.

${ }^{*}$ The authors acknowledge the support of: GNAMPA and GNCS groups of INdAM (Istituto Nazionale di Alta Matematica); MIUR-PRIN 2017, Project Stochastic Models for Complex Systems (no. 2017JFFHSH); MIUR Excellence Department Project awarded to the Department of Mathematics, University of Rome Tor Vergata (CUP E83C18000100006).

${ }^{\dagger}$ Dipartimento di Matematica, Università di Roma Tor Vergata, Via della Ricerca Scientifica, 00133 Rome, Italy. e-mail: macci@mat.uniroma2.it

${ }^{\ddagger}$ Dipartimento di Matematica, Università degli Studi di Salerno, Via Giovanni Paolo II n. 132, 84084 Fisciano, SA, Italy. e-mail: bmartinucci@unisa.it

${ }^{\S}$ Dipartimento di Matematica e Applicazioni, Università di Napoli Federico II, Via Cintia, Complesso Monte S. Angelo, 80126 Naples, Italy. e-mail: enrica.pirozzi@unina.it 
Now we describe the stochastic process studied in [5]. It represents a random motion of a particle on the half-line $[0, \infty)$. The particle moves up and down in an alternating way; moreover it has velocity 1 for the upward periods, and it has velocity -1 for the downward periods. Initially the motion proceeds upward for a positive random time $U_{1}$ and, after that, the particle moves downward for a positive random time $D_{1}$; moreover the motion alternates the random times $U_{2}, D_{2}, U_{3}, D_{3}, \ldots$, where $\left\{U_{n}: n \geq 1\right\}$ and $\left\{D_{n}: n \geq 1\right\}$ are independent sequences of i.i.d. positive random variables. Furthermore, when the particle hits the origin, it can be either absorbed or reflected upwards with probabilities $\alpha$ and $1-\alpha$, respectively (here $\alpha \in(0,1)$ but, actually, the case $\alpha=1$ is also allowed). More precisely, if during a downward period $D_{j}$, say, the particle reaches the origin and it is not absorbed, then instantaneously the particle starts an upward period for an independent random time $U_{j+1}$. We also remark that, here, we restrict our attention on the case in which the random variables $\left\{U_{n}: n \geq 1\right\}$ and $\left\{D_{n}: n \geq 1\right\}$ are exponentially distributed with parameters $\lambda$ and $\mu$, respectively; moreover we assume that $\lambda>\mu$ and this guarantees that $\mathbb{E}\left[D_{1}\right]>\mathbb{E}\left[U_{1}\right]$.

In particular we are interested in the random variable $A_{x}=A_{x}(\lambda, \mu)$, i.e. the absorption time of the particle when it starts at $x$; see equation (2) in [5]. The aim is to study the asymptotic behavior of that random variable with respect two different scalings:

- Scaling 1: $x \rightarrow \infty$;

- Scaling 2: $\mu \rightarrow \infty$, and $\lambda=\beta \mu$ for some fixed $\beta>1$ and $x>0$.

This will be done by referring to the theory of large deviations (see e.g. [2] as a reference on this topic). This theory allows to give an evaluation of probabilities of rare events on an exponential scale. Some preliminaries on this topic will be recalled in the next section 2 .

Some of these asymptotic results concern moderate deviations; this term is used in the literature when one has a class of large deviation principles which fills the gap between the convergence to a constant (typically governed by a large deviation principle) and an asymptotic Normality result (see Remarks 3.1 and 4.2). Interestingly in this paper we can also present a non-central moderate deviation result as $\mu \rightarrow \infty$ stated in Proposition 4.3 this means that we have a class of large deviation principles that fills the gap between the convergence to a constant and the weak convergence to a non-Gaussian limit (see Remark 4.4).

In this paper we also present some numerical estimates for $\beta$ (approximate confidence intervals and point estimations) obtained by simulations and based on an asymptotic Normality result as $\mu \rightarrow \infty$; moreover, as far as the scaling 1 is concerned, we also study the case in which the particle starts at some independent random point $Y(x)$.

We conclude with the outline of the paper. We start with some preliminaries in Section 2, The results are proved in Section 3 (for the scaling 1) and in Section 4 (for the scaling 2). Finally, in Section 5, we present the numerical estimates.

\section{Preliminaries}

We start with some preliminaries on large deviations. Then we conclude with some details on the model studied in [5]; actually we recall some preliminaries on the absorption time $A_{x}=A_{x}(\lambda, \mu)$.

\subsection{Preliminaries on large deviations}

We start with some basic definitions (see e.g. [2], pages 4-5). Let $\mathcal{Z}$ be a topological space equipped with its completed Borel $\sigma$-field. A family of $\mathcal{Z}$-valued random variables $\left\{Z_{r}: r>0\right\}$ (defined on the same probability space $(\Omega, \mathcal{F}, P)$ ) satisfies the large deviation principle (LDP for short) with speed function $v_{r}$ and rate function $I$ if: $\lim _{r \rightarrow \infty} v_{r}=\infty$; the function $I: \mathcal{Z} \rightarrow[0, \infty]$ is lower 
semi-continuous;

$$
\begin{aligned}
& \limsup _{n \rightarrow \infty} \frac{1}{v_{r}} \log P\left(Z_{r} \in F\right) \leq-\inf _{z \in F} I(z) \text { for all closed sets } F \\
& \liminf _{r \rightarrow \infty} \frac{1}{v_{r}} \log P\left(Z_{r} \in G\right) \geq-\inf _{z \in G} I(z) \text { for all open sets } G
\end{aligned}
$$

A rate function $I$ is said to be $\operatorname{good}$ if its level sets $\{\{z \in \mathcal{Z}: I(z) \leq \eta\}: \eta \geq 0\}$ are compact.

Throughout this paper we prove LDPs with $\mathcal{Z}=\mathbb{R}$. We recall the following known result for future use.

Theorem 2.1 (Gärtner Ellis Theorem (on $\mathbb{R})$; see e.g. Theorem 2.3.6 in $[2]$ ). Let $\left\{Z_{r}: r>0\right\}$ be a family of real valued random variables (defined on the same probability space $(\Omega, \mathcal{F}, P)$ ). Assume that the function $\Lambda: \mathbb{R} \rightarrow(-\infty, \infty]$ defined by

$$
\Lambda(s):=\lim _{r \rightarrow \infty} \frac{1}{v_{r}} \log \mathbb{E}\left[e^{v_{r} s Z_{r}}\right] \quad(\text { for all } s \in \mathbb{R})
$$

exists, and it is finite in a neighborhood of the origin $s=0$. Moreover let $\Lambda^{*}: \mathbb{R} \rightarrow[0, \infty]$ defined by

$$
\Lambda^{*}(z):=\sup _{s \in \mathbb{R}}\{s z-\Lambda(s)\} .
$$

Then: (1) holds with $I=\Lambda^{*}$; a weak form of (2) with $I=\Lambda^{*}$ holds, i.e.

$$
\liminf _{r \rightarrow \infty} \frac{1}{v_{r}} \log P\left(Z_{r} \in G\right) \geq-\inf _{z \in G \cap \mathcal{E}} \Lambda^{*}(z) \text { for all open sets } G
$$

where $\mathcal{E}$ is the set of exposed points of I (namely the points in which I is finite and strictly convex); if $\Lambda$ is essentially smooth and lower semi-continuous, then the LDP holds with good rate function $I=\Lambda^{*}$.

We also recall that $\Lambda$ in the above statement is essentially smooth (see e.g. Definition 2.3.5 in [2]) if the interior of the set $\mathcal{D}_{\Lambda}:=\{s \in \mathbb{R}: \Lambda(s)<\infty\}$ is non-empty, if it is differentiable throughout the interior of that set, and if it is a steep function (namely $\left|\Lambda^{\prime}(s)\right|$ tends to infinity when $s$ in the interior of $\mathcal{D}_{\Lambda}$ approaches any finite point of its boundary).

\subsection{Preliminaries on the model}

We start with a slight correction of in Proposition 9 in [5] for the moment generating function of the absorption time $A_{x}=A_{x}(\lambda, \mu)$. In what follows we consider the function

$$
\Lambda(s ; \lambda, \mu):=\frac{1}{2}\left(\lambda-\mu-\sqrt{(\lambda+\mu-2 s)^{2}-4 \lambda \mu}\right)
$$

for $s \leq \frac{(\sqrt{\lambda}-\sqrt{\mu})^{2}}{2}$. Throughout this paper we use the symbols $\Lambda^{\prime}(s ; \lambda, \mu)$ and $\Lambda^{\prime \prime}(s ; \lambda, \mu)$ for the first and the second derivatives of $\Lambda(s ; \lambda, \mu)$ with respect to $s$.

Remark 2.1 (The moment generating function of $A_{x}=A_{x}(\lambda, \mu)$ ). Proposition 9 in [5] provides an expression of the moment generating function $\mathbb{E}\left[e^{s A_{x}(\lambda, \mu)}\right]$ when $s \leq \frac{(\sqrt{\lambda}-\sqrt{\mu})^{2}}{2}$ (it is equal to infinity otherwise). Actually a possible further restriction on $s$ is needed, i.e. $(1-\alpha) \mathbb{E}\left[e^{s C_{0}}\right]<1$, where $\mathbb{E}\left[e^{s C_{0}}\right]$ is the moment generating function of the renewal cycles $\left\{C_{0, i}: i \geq 1\right\}$ introduced in [5]. Then, since $\mathbb{E}\left[e^{s C_{0}}\right] \uparrow \sqrt{\frac{\lambda}{\mu}}$ as $s \uparrow \frac{(\sqrt{\lambda}-\sqrt{\mu})^{2}}{2}$, we distinguish two cases: 
- if $(1-\alpha) \sqrt{\frac{\lambda}{\mu}}<1$ or, equivalently, if $\alpha>1-\sqrt{\frac{\mu}{\lambda}}$, then we recover the expression in Proposition 9 in [5], i.e.

$$
\mathbb{E}\left[e^{s A_{x}(\lambda, \mu)}\right]= \begin{cases}\frac{2 \alpha \lambda e^{x \Lambda(s ; \lambda, \mu)}}{2 \lambda(\alpha-1)+\lambda+\mu-2 s+\sqrt{(\lambda+\mu-2 s)^{2}-4 \lambda \mu}} & \text { for } s \leq \frac{(\sqrt{\lambda}-\sqrt{\mu})^{2}}{2} \\ \infty & \text { otherwise }\end{cases}
$$

- if $(1-\alpha) \sqrt{\frac{\lambda}{\mu}} \geq 1$ or, equivalently, if $\alpha \leq 1-\sqrt{\frac{\mu}{\lambda}}$, then we have

$$
\mathbb{E}\left[e^{s A_{x}(\lambda, \mu)}\right]= \begin{cases}\frac{2 \alpha \lambda e^{x \Lambda(s ; \lambda, \mu)}}{2 \lambda(\alpha-1)+\lambda+\mu-2 s+\sqrt{(\lambda+\mu-2 s)^{2}-4 \lambda \mu}} & \text { for } s<\hat{s}(\lambda, \mu, \alpha) \\ \infty & \text { otherwise, }\end{cases}
$$

where $\hat{s}(\lambda, \mu, \alpha):=\frac{\alpha(\lambda(1-\alpha)-\mu)}{2(1-\alpha)} \in\left(0, \frac{(\sqrt{\lambda}-\sqrt{\mu})^{2}}{2}\right]$; in particular we have $\hat{s}(\lambda, \mu, \alpha)=\frac{(\sqrt{\lambda}-\sqrt{\mu})^{2}}{2}$ if $(1-\alpha) \sqrt{\frac{\lambda}{\mu}}=1$ or, equivalently, if $\alpha=1-\sqrt{\frac{\mu}{\lambda}}$.

Now we discuss some technical details on the function $\Lambda(\cdot)=\Lambda(\cdot ; \lambda, \mu)$ (in particular the concept of steepness in the definition of essentially smooth function).

Remark 2.2 (Some properties of $\Lambda(\cdot ; \lambda, \mu)$ ). The function $\Lambda(\cdot)=\Lambda(\cdot ; \lambda, \mu)$ plays a crucial role in some applications of the Gärtner Ellis Theorem in this paper. In particular, by referring to Remark 2.1. it is a lower semi-continuous function if $\alpha>1-\sqrt{\frac{\mu}{\lambda}}$, and it is an essentially smooth function if $\alpha \geq 1-\sqrt{\frac{\mu}{\lambda}}$.

In view of some results presented below, we compute the following Legendre transforms:

$$
\Lambda^{*}(z ; \lambda, \mu):=\sup _{s \in \mathbb{R}}\{s z-\Lambda(s ; \lambda, \mu)\}=\sup _{s \leq \frac{(\sqrt{\lambda}-\sqrt{\mu})^{2}}{2}}\{s z-\Lambda(s ; \lambda, \mu)\}
$$

and, if we consider $\hat{s}(\lambda, \mu, \alpha)$ in Remark 2.1 for $\alpha \leq 1-\sqrt{\frac{\mu}{\lambda}}$,

$$
\Lambda^{*}(z ; \lambda, \mu, \alpha):=\sup _{s \leq \hat{s}(\lambda, \mu, \alpha)}\{s z-\Lambda(s ; \lambda, \mu)\} .
$$

Lemma 2.1 (Computation of Legendre transforms). We have

$$
\Lambda^{*}(z ; \lambda, \mu)= \begin{cases}\frac{1}{2}(\sqrt{(z-1) \lambda}-\sqrt{(z+1) \mu})^{2} & \text { if } z \geq 1 \\ \infty & \text { otherwise. }\end{cases}
$$

Moreover, for $\alpha \leq 1-\sqrt{\frac{\mu}{\lambda}}$, if we set $\tilde{z}(\lambda, \mu, \alpha):=\frac{\lambda+\mu-2 \hat{s}(\lambda, \mu, \alpha)}{\sqrt{(\lambda+\mu-2 \hat{s}(\lambda, \mu, \alpha))^{2}-4 \lambda \mu}}$ for $\hat{s}(\lambda, \mu, \alpha)$ as in Remark 2.1, then

$$
\begin{aligned}
& \Lambda^{*}(z ; \lambda, \mu, \alpha)= \begin{cases}\Lambda^{*}(z ; \lambda, \mu) \\
\hat{s}(\lambda, \mu, \alpha) z-\Lambda(\hat{s}(\lambda, \mu, \alpha) ; \lambda, \mu) & \text { if } z \leq \tilde{z}(\lambda, \mu, \alpha) \\
\text { otherwise }\end{cases} \\
& = \begin{cases}\infty & \text { if } z<1 \\
\frac{1}{2}(\sqrt{(z-1) \lambda}-\sqrt{(z+1) \mu})^{2} & \text { if } 1 \leq z \leq \tilde{z}(\lambda, \mu, \alpha) \\
\hat{s}(\lambda, \mu, \alpha) z-\Lambda(\hat{s}(\lambda, \mu, \alpha) ; \lambda, \mu) & \text { if } z>\tilde{z}(\lambda, \mu, \alpha) .\end{cases}
\end{aligned}
$$


Proof. We start with the first statement concerning $\Lambda^{*}(z ; \lambda, \mu)$. For $z>1$ one can check that the equation $z=\Lambda^{\prime}(s ; \lambda, \mu)$ has solution $s=s_{z}:=\frac{1}{2}\left(\lambda+\mu-2 z \sqrt{\frac{\lambda \mu}{z^{2}-1}}\right)$, and

$$
\Lambda^{*}(z ; \lambda, \mu)=s_{z} z-\Lambda\left(s_{z} ; \lambda, \mu\right)
$$

yields the desired expression; for $z \leq 1$ one can check that

$$
\Lambda^{*}(z ; \lambda, \mu)=\lim _{s \rightarrow-\infty} s z-\Lambda(s ; \lambda, \mu)
$$

which yields again the desired expression (one has to distinguish the cases $z=1$ and $z<1$ ).

For $\Lambda^{*}(z ; \lambda, \mu, \alpha)$ we proceed in the same way, and we omit some details. Some computations coincide with the ones presented above but, if $s_{z}$ above is larger than $\hat{s}(\lambda, \mu, \alpha)$, then the supremum is attained at $s=\hat{s}(\lambda, \mu, \alpha)$. Moreover one can check that $s_{z}>\hat{s}(\lambda, \mu, \alpha)$ if and only if $z>\tilde{z}(\lambda, \mu, \alpha)$. The desired expression can be checked with straightforward computations.

Finally, in the next Remark 2.3, we recall some formulas already presented in [5]; actually we give the corrected expression of the variance.

Remark 2.3 (A correction of a variance formula in [5]). Here we give the correct version of some formulas in [5]. More precisely we mean the $n$-th moments of $C_{x}$ and $A_{x}$, i.e.

$$
\begin{aligned}
& \mathbb{E}\left[C_{x}^{n}\right]=\frac{\lambda}{\lambda+\mu} \mathrm{e}^{\frac{x}{2}(\lambda-\mu)} \frac{2^{n} n !}{(\lambda+\mu)^{n}} \sum_{h=0}^{n}\left(-\frac{\lambda+\mu}{(\sqrt{\lambda}-\sqrt{\mu})^{2}}\right)^{h} \\
& \quad \times{ }_{2} F_{1}\left(\frac{1+n-h}{2}, \frac{2+n-h}{2} ; 2 ; \frac{4 \lambda \mu}{(\lambda+\mu)^{2}}\right) \\
& \quad \times \sum_{j=0}^{+\infty}\left[-\frac{(\lambda-\mu) x}{2}\right]^{j} \frac{1}{j !}\left(\begin{array}{c}
j / 2 \\
h
\end{array}\right){ }_{2} F_{1}\left(-h,-\frac{j}{2} ; \frac{j}{2}+1-h ;\left(\frac{\sqrt{\lambda}-\sqrt{\mu}}{\sqrt{\lambda}+\sqrt{\mu}}\right)^{2}\right),
\end{aligned}
$$

and

$$
\begin{aligned}
\mathbb{E}\left[A_{x}^{n}\right]=2 \alpha \lambda n ! \mathrm{e}^{\frac{x}{2}(\lambda-\mu)} \sum_{h=0}^{n}\left(-\frac{2}{(\sqrt{\lambda}-\sqrt{\mu})^{2}}\right)^{h} \frac{(8 \lambda(\alpha-1))^{n-h}}{(4 \lambda \alpha(\mu+\lambda(\alpha-1)))^{n-h+1}} \\
\times\left[2 \mu+2 \lambda(\alpha-1)+\frac{2 \lambda \mu}{\lambda+\mu} \sum_{m=1}^{n-h}\left(\frac{\alpha \mu+\alpha \lambda(\alpha-1)}{(\alpha-1)(\lambda+\mu)}\right)^{m}\right. \\
\left.\times{ }_{2} F_{1}\left(\frac{m+1}{2}, \frac{m+2}{2} ; 2 ; \frac{4 \lambda \mu}{(\lambda+\mu)^{2}}\right)\right] \\
\times \sum_{j=0}^{+\infty}\left[-\frac{(\lambda-\mu) x}{2}\right]^{j} \frac{1}{j !}\left(\begin{array}{c}
j / 2 \\
h
\end{array}\right){ }_{2} F_{1}\left(-h,-\frac{j}{2} ; \frac{j}{2}+1-h ;\left(\frac{\sqrt{\lambda}-\sqrt{\mu}}{\sqrt{\lambda}+\sqrt{\mu}}\right)^{2}\right) .
\end{aligned}
$$

In particular we also recall the correct expressions in Proposition 12 in [5] (actually only the variances should be corrected):

$$
\begin{gathered}
\mathbb{E}\left[C_{x}\right]=\frac{2+(\lambda+\mu) x}{\lambda-\mu} \text { and } \operatorname{Var}\left[C_{x}\right]=\frac{4(\lambda+\mu+2 \lambda \mu x)}{(\lambda-\mu)^{3}} ; \\
\mathbb{E}\left[A_{x}\right]=\frac{2+\alpha(\lambda+\mu) x}{\alpha(\lambda-\mu)} \text { and } \operatorname{Var}\left[A_{x}\right]=\frac{4\left(\lambda+2 \lambda \mu x \alpha^{2}+\mu(2 \alpha-1)\right)}{(\lambda-\mu)^{3} \alpha^{2}} .
\end{gathered}
$$




\section{Asymptotic results under the scaling 1}

We start with the standard large deviation result.

Proposition 3.1 (LD as $x \rightarrow \infty)$. Assume that $\alpha \geq 1-\sqrt{\frac{\mu}{\lambda}}$. Then the family $\left\{\frac{A_{x}}{x}: x>0\right\}$ satisfies the LDP with speed $x$, and good rate function $I_{1}$ defined by $I_{1}(z):=\Lambda^{*}(z ; \lambda, \mu)$.

Proof. It is easy to check (by taking into account Remark 2.1) that

$$
\left.\lim _{x \rightarrow \infty} \frac{1}{x} \log \mathbb{E}\left[e^{s A_{x}(\lambda, \mu)}\right]=\Lambda(s ; \lambda, \mu) \text { (for all } s \in \mathbb{R}\right) .
$$

Then, by taking into account Remark 2.2, the desired LDP holds by a straightforward application of the Gärtner Ellis Theorem.

Note that $\frac{A_{x}}{x}$ converges to $z_{1}:=\frac{\lambda+\mu}{\lambda-\mu}$ almost surely as $x \rightarrow \infty$ (this can be checked in a standard way noting that the rate function $I_{1}$ uniquely vanishes at $\left.z_{1}\right)$. Moreover $z_{1}=\Lambda^{\prime}(0 ; \lambda, \mu)=$ $\lim _{x \rightarrow \infty} \frac{\mathbb{E}\left[A_{x}\right]}{x}$. Finally $z_{1}$ can be seen as the abscissa of the intersection (in the $\tilde{x} \tilde{y}$ plane) of the lines $\tilde{y}=0$ and $\tilde{y}=1+\frac{\frac{1}{\lambda}-\frac{1}{\mu}}{\frac{1}{\lambda}+\frac{1}{\mu}} \tilde{x}$. A version of Proposition 3.1 concerning the case $\alpha<1-\sqrt{\frac{\mu}{\lambda}}$ will be illustrated in Remark 3.2 (case $r=1)$.

Now we present the moderate deviation result. As it typically happens, we have a class of LDPs governed by the same quadratic rate function (i.e. $\tilde{I}_{1}$ ). Moreover this class of LDPs fills the gap between a convergence to zero and a weak convergence to a centered Normal distribution; see Remark 3.1 for some details and comments.

Proposition 3.2 (MD as $x \rightarrow \infty)$. For every family of positive numbers $\left\{\varepsilon_{x}: x>0\right\}$ such that

$$
\varepsilon_{x} \rightarrow 0 \text { and } x \varepsilon_{x} \rightarrow \infty
$$

the family $\left\{\frac{A_{x}-\mathbb{E}\left[A_{x}\right]}{\sqrt{x / \varepsilon_{x}}}: x>0\right\}$ satisfies the LDP with speed $1 / \varepsilon_{x}$, and good rate function $\tilde{I}_{1}$ defined by $\tilde{I}_{1}(z):=\frac{z^{2}}{2 \Lambda^{\prime \prime}(0 ; \lambda, \mu)}$, where $\Lambda^{\prime \prime}(0 ; \lambda, \mu)=\frac{8 \lambda \mu}{(\lambda-\mu)^{3}}$.

Proof. It suffices to show that

$$
\lim _{x \rightarrow \infty} \frac{1}{1 / \varepsilon_{x}} \log \mathbb{E}\left[e^{\frac{s}{\varepsilon_{x}} \frac{A_{x}-\mathbb{E}\left[A_{x}\right]}{\sqrt{x / \varepsilon_{x}}}}\right]=\frac{\Lambda^{\prime \prime}(0 ; \lambda, \mu)}{2} s^{2}(\text { for all } s \in \mathbb{R}) ;
$$

in fact the limit is a finite and differentiable function (with respect to $s \in \mathbb{R}$ ) and, noting that

$$
\tilde{I}_{1}(z)=\sup _{s \in \mathbb{R}}\left\{s z-\frac{\Lambda^{\prime \prime}(0 ; \lambda, \mu)}{2} s^{2}\right\}(\text { for all } z \in \mathbb{R}),
$$

the desired LDP is a straightforward application of the Gärtner Ellis Theorem.

We remark that

$$
\frac{1}{1 / \varepsilon_{x}} \log \mathbb{E}\left[e^{\frac{s}{\varepsilon_{x}} \frac{A_{x}-\mathbb{E}\left[A_{x}\right]}{\sqrt{x / \varepsilon_{x}}}}\right]=\varepsilon_{x}\left(\log \mathbb{E}\left[e^{\frac{s}{\sqrt{x \varepsilon_{x}}} A_{x}}\right]-\frac{s}{\sqrt{x \varepsilon_{x}}} \mathbb{E}\left[A_{x}\right]\right)
$$

and, since $\frac{s}{\sqrt{x \varepsilon_{x}}}$ is close to zero for $x$ large enough, it is easy to check (by the expressions of the moment generating function in Remark 2.1 and by (3)) that

$$
\begin{aligned}
& \lim _{x \rightarrow \infty} \frac{1}{1 / \varepsilon_{x}} \log \mathbb{E}\left[e^{\frac{s}{\varepsilon_{x}} \frac{A_{x}-\mathbb{E}\left[A_{x}\right]}{\sqrt{x / \varepsilon_{x}}}}\right] \\
& =\lim _{x \rightarrow \infty} \varepsilon_{x}\left(x \Lambda\left(\frac{s}{\sqrt{x \varepsilon_{x}}} ; \lambda, \mu\right)-\frac{s}{\sqrt{x \varepsilon_{x}}} \mathbb{E}\left[A_{x}\right]\right)=\lim _{x \rightarrow \infty} x \varepsilon_{x}\left(\Lambda\left(\frac{s}{\sqrt{x \varepsilon_{x}}} ; \lambda, \mu\right)-\frac{s}{\sqrt{x \varepsilon_{x}}} \frac{\mathbb{E}\left[A_{x}\right]}{x}\right) .
\end{aligned}
$$


Now we take into account the Mac Laurin formula of order 2 for the function $\Lambda(\cdot ; \lambda, \mu)$, and we have

$$
\begin{aligned}
& \lim _{x \rightarrow \infty} \frac{1}{1 / \varepsilon_{x}} \log \mathbb{E}\left[e^{\left.\frac{s}{\varepsilon_{x}} \frac{A_{x}-\mathbb{E}\left[A_{x}\right]}{\sqrt{x / \varepsilon_{x}}}\right]}\right] \\
& \quad=\lim _{x \rightarrow \infty} x \varepsilon_{x}\left(\left(\Lambda^{\prime}(0 ; \lambda, \mu)-\frac{\mathbb{E}\left[A_{x}\right]}{x}\right) \frac{s}{\sqrt{x \varepsilon_{x}}}+\frac{\Lambda^{\prime \prime}(0 ; \lambda, \mu)}{2} \frac{s^{2}}{x \varepsilon_{x}}+o\left(\frac{s^{2}}{x \varepsilon_{x}}\right)\right)
\end{aligned}
$$

where, by the mean value in (4),

$$
\Lambda^{\prime}(0 ; \lambda, \mu)-\frac{\mathbb{E}\left[A_{x}\right]}{x}=\frac{\lambda+\mu}{\lambda-\mu}-\frac{2+\alpha(\lambda+\mu) x}{\alpha(\lambda-\mu) x}=-\frac{2}{\alpha(\lambda-\mu) x} ;
$$

thus

$$
\begin{gathered}
\lim _{x \rightarrow \infty} \frac{1}{1 / \varepsilon_{x}} \log \mathbb{E}\left[e^{\frac{s}{\varepsilon_{x}} \frac{A_{x}-\mathbb{E}\left[A_{x}\right]}{\sqrt{x / \varepsilon_{x}}}}\right]=\lim _{x \rightarrow \infty}-\frac{2 s \sqrt{x \varepsilon_{x}}}{\alpha(\lambda-\mu) x}+\frac{\Lambda^{\prime \prime}(0 ; \lambda, \mu)}{2} s^{2}+x \varepsilon_{x} O\left(\frac{s^{2}}{x \varepsilon_{x}}\right) \\
=\frac{\Lambda^{\prime \prime}(0 ; \lambda, \mu)}{2} s^{2}+\lim _{x \rightarrow \infty}-\frac{2 s \sqrt{\varepsilon_{x}}}{\alpha(\lambda-\mu) \sqrt{x}}+x \varepsilon_{x} o\left(\frac{s^{2}}{x \varepsilon_{x}}\right)=\frac{\Lambda^{\prime \prime}(0 ; \lambda, \mu)}{2} s^{2} .
\end{gathered}
$$

Remark 3.1 (Typical features on MD in Proposition 3.2). The class of LDPs in Proposition 3.2 fills the gap between the two following asymptotic regimes as $x \rightarrow \infty$ :

- the convergence to zero of $\frac{A_{x}-\mathbb{E}\left[A_{x}\right]}{x}\left(\right.$ case $\left.\varepsilon_{\mu}=1 / x\right)$;

- the weak convergence of $\frac{A_{x}-\mathbb{E}\left[A_{x}\right]}{\sqrt{x}}$ to the centered Normal distribution with variance $\Lambda^{\prime \prime}(0 ; \lambda, \mu)$ (case $\varepsilon_{x}=1$ ).

In both cases one condition in (5) holds, and the other one fails. We also note that, by taking into account the variance expression in (4), we have $\Lambda^{\prime \prime}(0 ; \lambda, \mu)=\lim _{x \rightarrow \infty} \frac{\operatorname{Var}\left[A_{x}\right]}{x}$.

We conclude this section by considering a generalization of Proposition 3.1 with an independent random perturbation $Y(x)$ of the initial state $x$ under suitable hypotheses collected in Condition 1 . below; this generalization will be given in Proposition 3.4 and it will be followed by some remarks and comments. We start with the following slight generalization of Proposition 3.1 where the initial state is modified in a deterministic way; we recover the case in that proposition by setting $r=1$.

Proposition 3.3 (Slight extension of Proposition 3.1. Assume that $\alpha \geq 1-\sqrt{\frac{\mu}{\lambda}}$ and let $r>0$ be arbitrarily fixed. Then the family $\left\{\frac{A_{r x}}{x}: x>0\right\}$ satisfies the LDP with speed $x$, and good rate function $I_{1}(\cdot ; r)$ defined by $I_{1}(z ; r):=r \Lambda^{*}(z / r ; \lambda, \mu)$.

Proof. It is easy to check that

$$
\lim _{x \rightarrow \infty} \frac{1}{x} \log \mathbb{E}\left[e^{s A_{r x}(\lambda, \mu)}\right]=r \Lambda(s ; \lambda, \mu)(\text { for all } s \in \mathbb{R})
$$

(it is a slight modification of the analogue limit in the proof of Proposition 3.1 where $r=1$ ). Then, by taking into account Remark 2.2, the desired LDP holds by a straightforward application of the Gärtner Ellis Theorem. In fact the governing rate function $I_{1}(\cdot ; r)$ is defined by

$$
I_{1}(z ; r):=\sup _{s \in \mathbb{R}}\{s z-r \Lambda(s ; \lambda, \mu)\}=r \sup _{s \in \mathbb{R}}\{s z / r-\Lambda(s ; \lambda, \mu)\},
$$

and this coincides with the rate function in the statement of the proposition. 
Arguing as we did just after Proposition 3.1 , we can say that $\frac{A_{r x}}{x}$ converges to $r z_{1}=r \frac{\lambda+\mu}{\lambda-\mu}$ almost surely as $x \rightarrow \infty$ (and the rate function $I_{1}(\cdot ; r)$ uniquely vanishes at $r z_{1}$ ).

Remark 3.2 (Versions of Propositions 3.1 and 3.3 with exposed points). Here we discuss what happens when we consider the inequality $\alpha<1-\sqrt{\frac{\mu}{\lambda}}$ in Proposition 3.3 (and therefore in Proposition 3.1 for the case $r=1$ ). We have to consider some items of in the second part of Lemma 2.1 and, by the Gärtner Ellis Theorem, we have

$$
\limsup _{x \rightarrow \infty} \frac{1}{x} \log P\left(\frac{A_{r x}}{x} \in F\right) \leq-\inf _{z \in F} r \Lambda^{*}(z / r ; \lambda, \mu, \alpha) \text { for all closed sets } F
$$

and

$$
\liminf _{x \rightarrow \infty} \frac{1}{x} \log P\left(\frac{A_{r x}}{x} \in G\right) \geq-\inf _{z \in G \cap \mathcal{E}} r \Lambda^{*}(z / r ; \lambda, \mu, \alpha) \text { for all open sets } G
$$

where $\mathcal{E}=(r \tilde{z}(\lambda, \mu, \alpha), \infty)$ is the set of exposed points of $r \Lambda^{*}(\cdot / r ; \lambda, \mu, \alpha)$. Note that $r \tilde{z}(\lambda, \mu, \alpha)>$ $r z_{1}$, and therefore both $r \Lambda^{*}(\cdot / r ; \lambda, \mu, \alpha)$ and $I_{1}(\cdot ; r)$ uniquely vanish at $r z_{1}$.

Now we introduce the condition on the random perturbation of the initial state.

Condition 1. Let $\{Y(x): x \geq 0\}$ be a family of nonnegative random variables and assume that there exists the function $\Psi_{Y}: \mathbb{R} \rightarrow(-\infty, \infty]$ such that

$$
\Psi_{Y}(s):=\lim _{x \rightarrow \infty} \frac{1}{x} \log \mathbb{E}\left[e^{s Y(x)}\right]
$$

for all $s \in \mathbb{R}$. The function $\Psi_{Y}$ is nondecreasing by construction; so we consider the set

$$
\mathcal{D}_{Y}:=\left\{s \in \mathbb{R}: \Psi_{Y}(s)<\infty\right\},
$$

and we assume that either $\mathcal{D}_{Y}=\mathbb{R}$ or, for some $\bar{s}>0, \mathcal{D}_{Y}=(-\infty, \bar{s})$ or $\mathcal{D}_{Y}=(-\infty, \bar{s}]$ (note that $\left.(-\infty, 0] \subset \mathcal{D}_{Y}\right)$.

We remark that Condition 1 holds when $\{Y(x): x \geq 0\}$ belongs to a wide class of nondecreasing (with respect to $x$ ) Lévy processes, also called subordinators; in this case we have $\Psi_{Y}(s):=\log \mathbb{E}\left[e^{s Y(1)}\right]$. For instance we recall the following examples of infinitely divisible distributions concerning the random variable $Y(1)$.

$\begin{array}{cccccc}\text { Distribution } & \text { parameters } & \mathcal{D}_{Y} & \Psi_{Y}(s) \text { for } s \in \mathcal{D}_{Y} & \Psi_{Y}^{\prime}(0) & \Psi_{Y}^{\prime \prime}(0) \\ \text { Poisson } & \lambda>0 & \mathbb{R} & \lambda\left(e^{s}-1\right) & \lambda & \lambda \\ \text { Gamma } & \lambda, \theta>0 & (-\infty, \bar{s}), \bar{s}=\theta & \gamma \log \frac{\theta}{\theta-s} & \frac{\lambda}{\theta} & \frac{\lambda}{\theta^{2}} \\ \text { Inverse Gaussian } & \xi>0 & (-\infty, \bar{s}], \bar{s}=\frac{\xi^{2}}{2} & \xi-\sqrt{\xi^{2}-2 s} & \xi^{-1} & \xi^{-3}\end{array}$

So now we are ready to state the main generalization of Proposition 3.1

Proposition 3.4 (Extension of Proposition 3.1. Assume that $\alpha \geq 1-\sqrt{\frac{\mu}{\lambda}}$ and that a process $\{Y(x): x \geq 0\}$, independent of $\left\{A_{x}: x \geq 0\right\}$, satisfies Condition 1. Moreover let $\Lambda_{Y}$ be the function defined by

$$
\Lambda_{Y}(s):= \begin{cases}\Psi_{Y}(\Lambda(s ; \lambda, \mu)) & \text { for } \Lambda(s ; \lambda, \mu) \in \mathcal{D}_{Y} \text { and } s \leq \frac{(\sqrt{\lambda}-\sqrt{\mu})^{2}}{2} \\ \infty & \text { otherwise, }\end{cases}
$$

and assume that it is essentially smooth. Then the family $\left\{\frac{A_{Y(x)}}{x}: x>0\right\}$ satisfies the LDP with speed $x$, and good rate function $\Lambda_{Y}^{*}$ defined by $\Lambda_{Y}^{*}(z):=\sup _{s \in \mathbb{R}}\left\{s z-\Lambda_{Y}(s)\right\}$. 
Proof. We compute the moment generating function of $A_{Y(x)}$ by considering the well-known equality $\mathbb{E}\left[e^{s A_{Y(x)}(\lambda, \mu)}\right]=\mathbb{E}\left[\left.\mathbb{E}\left[e^{s A_{r}(\lambda, \mu)}\right]\right|_{r=Y(x)}\right]$. Moreover, by the expression of the moment generating function in Remark 2.1, we get

$$
\mathbb{E}\left[e^{s A_{Y(x)}(\lambda, \mu)}\right]= \begin{cases}\frac{2 \alpha \lambda \mathbb{E}\left[e^{Y(x) \Lambda(s ; \lambda, \mu)}\right]}{2 \lambda(\alpha-1)+\lambda+\mu-2 s+\sqrt{(\lambda+\mu-2 s)^{2}-4 \lambda \mu}} & \text { for } s \leq \frac{(\sqrt{\lambda}-\sqrt{\mu})^{2}}{2} \\ \infty & \text { otherwise. }\end{cases}
$$

So, by Condition 1, we get

$$
\lim _{x \rightarrow \infty} \frac{1}{x} \log \mathbb{E}\left[e^{s A_{Y(x)}(\lambda, \mu)}\right]=\Lambda_{Y}(s)(\text { for all } s \in \mathbb{R}),
$$

where $\Lambda_{Y}$ is the function in the statement of the proposition. In conclusion, since $\Lambda_{Y}$ is an essentially smooth function, the desired LDP holds by a straightforward application of the Gärtner Ellis Theorem.

Now we present some remarks and comments on Proposition 3.4. In what follows we assume that the function $\Psi_{Y}$ is differentiable in the interior of $\mathcal{D}_{Y}$.

Remark 3.3 (Extension of some parts in the proof of Lemma 2.1). We consider $\Psi_{Y}^{\prime}(-\infty):=$ $\lim _{s \rightarrow-\infty} \Psi_{Y}^{\prime}(s)$ (this limit is well-defined because $\Psi_{Y}^{\prime}$ is monotonic by the convexity of $\left.\Psi_{Y}\right)$. Then, for $z>\Psi_{Y}^{\prime}(-\infty)$, one can check that the equation $z=\Lambda_{Y}^{\prime}(s)$ has solution $s=\tilde{s}_{z}$, and we have

$$
\Lambda_{Y}^{*}(z)=z \tilde{s}_{z}-\Lambda_{Y}\left(\tilde{s}_{z}\right) .
$$

On the other hand, for $z \leq \Psi_{Y}^{\prime}(-\infty)$ one can check that

$$
\Lambda_{Y}^{*}(z)=\lim _{s \rightarrow-\infty} s z-\Lambda_{Y}(s),
$$

which is finite for $z=\Psi_{Y}^{\prime}(-\infty)$ and infinite for $z<\Psi_{Y}^{\prime}(-\infty)$.

Remark 3.4 (On the essential smoothness of $\Lambda_{Y}$ ). In general, if s belongs to the interior of the set where $\Lambda_{Y}(s)<\infty$, we have

$$
\Lambda_{Y}^{\prime}(s)=\Psi_{Y}^{\prime}(\Lambda(s ; \lambda, \mu)) \Lambda^{\prime}(s ; \lambda, \mu) .
$$

Then, if we refer to Condition 1, we have two cases.

1. If $\mathcal{D}_{Y}=\mathbb{R}$, then we have to check that $\Lambda_{Y}^{\prime}(s) \uparrow \infty$ as $s \uparrow \frac{(\sqrt{\lambda}-\sqrt{\mu})^{2}}{2}$. This statement is true because $\Lambda^{\prime}(s ; \lambda, \mu) \uparrow \infty$ and $\Psi_{Y}^{\prime}(\Lambda(s ; \lambda, \mu))$ tends to a positive limit.

2. If we have $\mathcal{D}_{Y}=(-\infty, \bar{s})$ or $\mathcal{D}_{Y}=(-\infty, \bar{s}]$ for some $\bar{s} \in(0, \infty)$, then we take $s_{0}:=$ $\Lambda^{-1}(\bar{s} ; \lambda, \mu) \wedge \frac{(\sqrt{\lambda}-\sqrt{\mu})^{2}}{2}$ and we have to check that $\Lambda_{Y}^{\prime}(s) \uparrow \infty$ as $s \uparrow s_{0}$. If $s_{0}=\frac{(\sqrt{\lambda}-\sqrt{\mu})^{2}}{2}$, then we can conclude following the lines of the previous case $\left(\mathcal{D}_{Y}=\mathbb{R}\right)$. If $s_{0}=\Lambda^{-1}(\bar{s} ; \lambda, \mu)$, we also require the condition $\Psi_{Y}^{\prime}(s) \uparrow \infty$ as $s \uparrow \bar{s}$, and then we have $\Lambda_{Y}^{\prime}(s) \uparrow \infty$ because $\Psi_{Y}^{\prime}(\Lambda(s ; \lambda, \mu)) \uparrow \infty$ and $\Lambda^{\prime}(s ; \lambda, \mu)$ tends to a positive limit.

We continue with some further comments and, from now on, we assume that $\Psi_{Y}^{\prime}(0)>0$; note that this condition holds for the examples tabulated above. Moreover we assume to have the hypotheses of Propositions 3.3 and 3.4 that guarantee the validity of the LDPs stated in those propositions. It is known that $\Lambda_{Y}^{*}(z)=0$ if and only if $z=\hat{z}:=\Lambda_{Y}^{\prime}(0)=\Psi_{Y}^{\prime}(0) \Lambda^{\prime}(0 ; \lambda, \mu)$, and $I_{1}(z ; r)=0$ if and only if $z=z_{r}^{*}:=r \Lambda^{\prime}(0 ; \lambda, \mu)$. So, if we take $r=\Psi_{Y}^{\prime}(0)$, we have $\hat{z}=z_{r}^{*}$, both rate functions $\Lambda_{Y}^{*}$ and $I_{1}(\cdot ; r)$ uniquely vanish at $\hat{z}$, and therefore both $\frac{A_{Y(x)}}{x}$ and $\frac{A_{r x}}{x}$ converge to same 
limit $\hat{z}($ as $x \rightarrow \infty)$. Thus, in this case, it is interesting to find inequalities between rate functions (when $z$ belongs to a neighborhood of $\hat{z}$, except $z=\hat{z}$ ) to say that we have a faster convergence in the case governed by the (locally) larger rate function.

We start noting that $\Psi_{Y}(s) \geq \Psi_{Y}^{\prime}(0) s$ by the convexity of $\Psi_{Y}$ and by $\Psi_{Y}(0)=0$; thus we obtain

$$
\Lambda_{Y}(s) \geq \Psi_{Y}^{\prime}(0) \Lambda(s ; \lambda, \mu) \text { for all } s \in \mathbb{R}
$$

(in fact, if $s>\frac{(\sqrt{\lambda}-\sqrt{\mu})^{2}}{2}$, we have $\Lambda_{Y}(s)=\Psi_{Y}^{\prime}(0) \Lambda(s ; \lambda, \mu)=\infty$ ). So we easily obtain the following inequality between rate functions:

$$
\Lambda_{Y}^{*}(z)=\sup _{s \in \mathbb{R}}\left\{s z-\Lambda_{Y}(s)\right\} \leq \sup _{s \in \mathbb{R}}\left\{s z-\Psi_{Y}^{\prime}(0) \Lambda(s ; \lambda, \mu)\right\}=I_{1}\left(z ; \Psi_{Y}^{\prime}(0)\right) .
$$

In conclusion the rate function which governs the $\operatorname{LDP}$ of $\left\{\frac{A_{\Psi_{Y}^{\prime}(0) x}}{x}: x>0\right\}$ cannot be smaller than the one for the LDP of $\left\{\frac{A_{Y(x)}}{x}: x>0\right\}$; this is not surprising because we expect to have a faster convergence (to $\hat{z}$ as $x \rightarrow \infty$ ) when the perturbation of the initial position is deterministic.

We also remark that, under suitable conditions (for instance if $\Psi_{Y}$ is strictly convex, as happens for the tabulated examples above), we have the strict inequality $\Lambda_{Y}^{*}(z)<I_{1}\left(z ; \Psi_{Y}^{\prime}(0)\right)$ except for the cases in which both $\Lambda_{Y}^{*}(z)$ and $I_{1}\left(z ; \Psi_{Y}^{\prime}(0)\right)$ are equal to zero (i.e. if $\left.z=\hat{z}\right)$ or to infinity (i.e. if $\left.z<\Psi_{Y}^{\prime}(-\infty)\right)$.

As a final comment we also briefly discuss the comparison between the second derivatives of the rate functions at $z=\hat{z}$; indeed a larger second derivative corresponds to a locally larger rate function in a neighborhood of $\hat{z}$, except $z=\hat{z}$. We have

$$
\left(\Lambda_{Y}^{*}\right)^{\prime \prime}\left(\Lambda_{Y}^{\prime}(0)\right)=\frac{1}{\Lambda_{Y}^{\prime \prime}(0)}=\frac{1}{\Psi_{Y}^{\prime \prime}(0)\left(\Lambda^{\prime}(0 ; \lambda, \mu)\right)^{2}+\Psi_{Y}^{\prime}(0) \Lambda^{\prime \prime}(0 ; \lambda, \mu)}
$$

and

$$
I_{1}^{\prime \prime}\left(r \Lambda^{\prime}(0 ; \lambda, \mu) ; r\right)=\frac{1}{r \Lambda^{\prime \prime}(0 ; \lambda, \mu)}
$$

thus, if we set $r=\Psi_{Y}^{\prime}(0)$ in the last equalities, we get

$$
\left(\Lambda_{Y}^{*}\right)^{\prime \prime}(\hat{z}) \leq I_{1}^{\prime \prime}\left(\hat{z} ; \Psi_{Y}^{\prime}(0)\right)
$$

by the convexity of the function $\Psi_{Y}$ which yields $\Psi_{Y}^{\prime \prime}(0) \geq 0$. Actually in several common cases we have the strict inequality $\left(\Lambda_{Y}^{*}\right)^{\prime \prime}(\hat{z})<I_{1}^{\prime \prime}\left(\hat{z} ; \Psi_{Y}^{\prime}(0)\right)$ because $\Lambda^{\prime}(0 ; \lambda, \mu)>0$ and, as happens for the tabulated examples above, $\Psi_{Y}^{\prime \prime}(0)>0$.

\section{Asymptotic results under the scaling 2}

Throughout this section we set $\lambda=\beta \mu$ for some $\beta>1$ and $x>0$. We start with the standard large deviation result.

Proposition 4.1 $(\mathrm{LD}$ as $\mu \rightarrow \infty)$. Assume that $\alpha \geq 1-\sqrt{\frac{1}{\beta}}$. Then the family $\left\{A_{x}(\beta \mu, \mu): \mu>0\right\}$ satisfies the $L D P$ with speed $\mu$, and good rate function $I_{2}$ defined by $I_{2}(z):=x \Lambda^{*}(z / x ; \beta, 1)$.

Proof. It is easy to check (by taking into account Remark 2.1) that

$$
\lim _{\mu \rightarrow \infty} \frac{1}{\mu} \log \mathbb{E}\left[e^{\mu s A_{x}(\beta \mu, \mu)}\right]=x \Lambda(s ; \beta, 1)(\text { for all } s \in \mathbb{R}) .
$$


Then, by taking into account Remark 2.2, the desired LDP holds by a straightforward application of the Gärtner Ellis Theorem. In fact the governing rate function $I_{2}$ is defined by

$$
I_{2}(z):=\sup _{s \in \mathbb{R}}\{s z-x \Lambda(s ; \beta, 1)\}=x \sup _{s \in \mathbb{R}}\{s z / x-\Lambda(s ; \beta, 1)\},
$$

and this coincides with the rate function in the statement of the proposition.

Note that $A_{x}(\beta \mu, \mu)$ converges to $z_{2}:=x \frac{\beta+1}{\beta-1}$ almost surely as $\mu \rightarrow \infty$ (in fact the rate function $I_{2}$ uniquely vanishes at $\left.z_{2}\right)$. Moreover $z_{2}=x \Lambda^{\prime}(0 ; \beta, 1)=\lim _{\mu \rightarrow \infty} \mathbb{E}\left[A_{x}(\beta \mu, \mu)\right]$. Finally $z_{2}$ can be seen as the abscissa of the intersection (in the $\tilde{x} \tilde{y}$ plane) of the lines $\tilde{y}=0$ and $\tilde{y}=x+\frac{\frac{1}{\beta \mu}-\frac{1}{\mu}}{\frac{1}{\beta \mu}} \tilde{\frac{1}{\mu}} \tilde{x}$.

Remark 4.1 (A version of Proposition 4.1 with exposed points). Here we discuss what happens when we consider the inequality $\alpha<1-\sqrt{\frac{1}{\beta}}$ in Proposition 4.1. In this case we still have to consider some items of in the second part of Lemma 2.1 (as in Remark 3.2) and, by the Gärtner Ellis Theorem, we have

$$
\limsup _{\mu \rightarrow \infty} \frac{1}{\mu} \log P\left(A_{x}(\beta \mu, \mu) \in F\right) \leq-\inf _{z \in F} x \Lambda^{*}(z / x ; \beta, 1, \alpha) \text { for all closed sets } F
$$

and

$$
\liminf _{\mu \rightarrow \infty} \frac{1}{\mu} \log P\left(A_{x}(\beta \mu, \mu) \in G\right) \geq-\inf _{z \in G \cap \mathcal{E}} x \Lambda^{*}(z / x ; \beta, 1, \alpha) \text { for all open sets } G
$$

where $\mathcal{E}=(x \tilde{z}(\beta, 1, \alpha), \infty)$ is the set of exposed points of $x \Lambda^{*}(\cdot / x ; \beta, 1, \alpha)$. Note that $x \tilde{z}(\beta, 1, \alpha)>$ $z_{2}$, and therefore both $x \Lambda^{*}(\cdot / x ; \beta, 1, \alpha)$ and $I_{2}$ uniquely vanish at $z_{2}$.

Now we present the moderate deviation result. As it typically happens, we have a class of LDPs governed by the same quadratic rate function (i.e. $\tilde{I}_{2}$ ). Moreover this class of LDPs fills the gap between a convergence to zero and a weak convergence to a centered Normal distribution; see Remark 4.2 for some details and comments.

Proposition $4.2(\mathrm{MD}$ as $\mu \rightarrow \infty)$. For every family of positive numbers $\left\{\varepsilon_{\mu}: \mu>0\right\}$ such that

$$
\varepsilon_{\mu} \rightarrow 0 \text { and } \mu \varepsilon_{\mu} \rightarrow \infty
$$

the family $\left\{\sqrt{\mu \varepsilon_{\mu}}\left(A_{x}(\beta \mu, \mu)-\mathbb{E}\left[A_{x}(\beta \mu, \mu)\right]\right): \mu>0\right\}$ satisfies the LDP with speed $1 / \varepsilon_{\mu}$, and good rate function $\tilde{I}_{2}$ defined by $\tilde{I}_{2}(z):=\frac{z^{2}}{2 x \Lambda^{\prime \prime}(0 ; \beta, 1)}$, where $\Lambda^{\prime \prime}(0 ; \beta, 1)=\frac{8 \beta}{(\beta-1)^{3}}$.

Proof. It suffices to show that

$$
\lim _{\mu \rightarrow \infty} \frac{1}{1 / \varepsilon_{\mu}} \log \mathbb{E}\left[e^{\frac{s}{\varepsilon_{\mu}} \sqrt{\mu \varepsilon_{\mu}}\left(A_{x}(\beta \mu, \mu)-\mathbb{E}\left[A_{x}(\beta \mu, \mu)\right]\right)}\right]=\frac{x \Lambda^{\prime \prime}(0 ; \beta, 1)}{2} s^{2}(\text { for all } s \in \mathbb{R})
$$

in fact the limit is a finite and differentiable function (with respect to $s \in \mathbb{R}$ ) and, noting that

$$
\tilde{I}_{2}(z)=\sup _{s \in \mathbb{R}}\left\{s z-\frac{x \Lambda^{\prime \prime}(0 ; \beta, 1)}{2} s^{2}\right\}(\text { for all } z \in \mathbb{R})
$$

the desired LDP is a straightforward application of the Gärtner Ellis Theorem.

We remark that

$$
\frac{1}{1 / \varepsilon_{\mu}} \log \mathbb{E}\left[e^{\frac{s}{\varepsilon_{\mu}} \sqrt{\mu \varepsilon_{\mu}}\left(A_{x}(\beta \mu, \mu)-\mathbb{E}\left[A_{x}(\beta \mu, \mu)\right]\right)}\right]=\varepsilon_{\mu}\left(\log \mathbb{E}\left[e^{s \frac{\sqrt{\mu}}{\sqrt{\varepsilon_{\mu}}} A_{x}(\beta \mu, \mu)}\right]-\frac{s \sqrt{\mu}}{\sqrt{\varepsilon_{\mu}}} \mathbb{E}\left[A_{x}(\beta \mu, \mu)\right]\right) ;
$$


moreover

$$
\log \mathbb{E}\left[e^{s \frac{\sqrt{\mu}}{\sqrt{\varepsilon \mu}} A_{x}(\beta \mu, \mu)}\right]=\log \mathbb{E}\left[e^{\frac{s \mu}{\sqrt{\mu \varepsilon \mu}} A_{x}(\beta \mu, \mu)}\right]=\log \mathbb{E}\left[e^{\frac{s}{\sqrt{\mu \varepsilon \mu}} A_{x \mu}(\beta, 1)}\right]
$$

where the last equality holds by the expressions of the moment generating function in Remark 2.1 and by $(3)$; then, since $\frac{s}{\sqrt{\mu \varepsilon_{\mu}}}$ is close to zero for $\mu$ large enough, it is easy to check that

$$
\begin{aligned}
& \lim _{\mu \rightarrow \infty} \frac{1}{1 / \varepsilon_{\mu}} \log \mathbb{E}\left[e^{\left.\frac{s}{\varepsilon_{\mu}} \sqrt{\mu \varepsilon_{\mu}}\left(A_{x}(\beta \mu, \mu)-\mathbb{E}\left[A_{x}(\beta \mu, \mu)\right]\right)\right]}\right. \\
&=\lim _{\mu \rightarrow \infty} \varepsilon_{\mu}\left(x \mu \Lambda\left(\frac{s}{\sqrt{\mu \varepsilon_{\mu}}} ; \beta, 1\right)-\frac{s \mu}{\sqrt{\mu \varepsilon_{\mu}}} \mathbb{E}\left[A_{x}(\beta \mu, \mu)\right]\right) \\
&=\lim _{\mu \rightarrow \infty} \mu \varepsilon_{\mu}\left(x \Lambda\left(\frac{s}{\sqrt{\mu \varepsilon_{\mu}}} ; \beta, 1\right)-\frac{s}{\sqrt{\mu \varepsilon_{\mu}}} \mathbb{E}\left[A_{x}(\beta \mu, \mu)\right]\right) .
\end{aligned}
$$

Now we take into account the Mac Laurin formula of order 2 for the function $\Lambda(\cdot ; \beta, 1)$, and we have

$$
\begin{aligned}
& \lim _{\mu \rightarrow \infty} \frac{1}{1 / \varepsilon_{\mu}} \log \mathbb{E}\left[e^{\left.\frac{s}{\varepsilon_{\mu}} \sqrt{\mu \varepsilon_{\mu}}\left(A_{x}(\beta \mu, \mu)-\mathbb{E}\left[A_{x}(\beta \mu, \mu)\right]\right)\right]}\right. \\
& \quad=\lim _{\mu \rightarrow \infty} \mu \varepsilon_{\mu}\left(\left(x \Lambda^{\prime}(0 ; \beta, 1)-\mathbb{E}\left[A_{x}(\beta \mu, \mu)\right]\right) \frac{s}{\sqrt{\mu \varepsilon_{\mu}}}+\frac{x \Lambda^{\prime \prime}(0 ; \beta, 1)}{2} \frac{s^{2}}{\mu \varepsilon_{\mu}}+o\left(\frac{s^{2}}{\mu \varepsilon_{\mu}}\right)\right)
\end{aligned}
$$

where, by the mean value in (4),

$$
x \Lambda^{\prime}(0 ; \beta, 1)-\mathbb{E}\left[A_{x}(\beta \mu, \mu)\right]=x \frac{\beta+1}{\beta-1}-\frac{2+\alpha(\beta+1) \mu x}{\alpha(\beta-1) \mu}=-\frac{2}{\alpha(\beta-1) \mu}
$$

thus

$$
\begin{aligned}
\lim _{\mu \rightarrow \infty} \frac{1}{1 / \varepsilon_{\mu}} \log \mathbb{E}\left[e^{\left.\frac{s}{\varepsilon_{\mu}} \sqrt{\mu \varepsilon_{\mu}}\left(A_{x}(\beta \mu, \mu)-\mathbb{E}\left[A_{x}(\beta \mu, \mu)\right]\right)\right]}\right. & \\
= & \frac{x \Lambda^{\prime \prime}(0 ; \beta, 1)}{2} s^{2}+\lim _{\mu \rightarrow \infty}-\frac{2 \sqrt{\varepsilon_{\mu}}}{\alpha(\beta-1) \sqrt{\mu}} s+\mu \varepsilon_{\mu} O\left(\frac{s^{2}}{\mu \varepsilon_{\mu}}\right)=\frac{x \Lambda^{\prime \prime}(0 ; \beta, 1)}{2} s^{2} .
\end{aligned}
$$

Remark 4.2 (Typical features on MD in Proposition 4.2). The class of LDPs in Proposition 4.2 fills the gap between the two following asymptotic regimes as $\mu \rightarrow \infty$ :

- the convergence to zero of $A_{x}(\beta \mu, \mu)-\mathbb{E}\left[A_{x}(\beta \mu, \mu)\right]\left(\right.$ case $\left.\varepsilon_{\mu}=1 / \mu\right)$;

- the weak convergence of $\sqrt{\mu}\left(A_{x}(\beta \mu, \mu)-\mathbb{E}\left[A_{x}(\beta \mu, \mu)\right]\right)$ to the centered Normal distribution with variance $x \Lambda^{\prime \prime}(0 ; \beta, 1)$ (case $\left.\varepsilon_{\mu}=1\right)$.

In both cases one condition in (6) holds, and the other one fails. We also note that, by taking into account the variance expression in (4), we have $x \Lambda^{\prime \prime}(0 ; \beta, 1)=\lim _{\mu \rightarrow \infty} \mu \operatorname{Var}\left[A_{x}(\beta \mu, \mu)\right]$.

We conclude this section with another moderate deviation result, which will be stated in Proposition 4.3. Namely we mean a class of LDPs that fills the gap between two asymptotic regimes, as $\mu \rightarrow \infty$, as in Proposition 4.2, more precisely the convergence to a constant, and the weak convergence to a suitable non degenerate limit law (this will be explained in Remark 4.4 below). In some sense we have a non-central moderate deviation result because the limit law is non-Gaussian; actually, as shown in the next Lemma 4.1. we deal with a family of equally distributed random variables and therefore the weak convergence trivially holds. 
Lemma 4.1 (A weak convergence result as $\mu \rightarrow \infty)$. The random variables $\left\{\mu A_{x / \mu}(\beta \mu, \mu): \mu>0\right\}$ are equally distributed.

Proof. The result can be easily proved by taking the moment generating function of the involved random variables, and by referring to the formulas presented in Remark 2.1. One can easily check (we omit the details) that, under every condition on $\alpha$ stated in Remark 2.1, we have the same moment generating function for every random variables of the family $\left\{\mu A_{x / \mu}(\beta \mu, \mu): \mu>0\right\}$ (in fact it does not depend on $\mu$ ).

Now we can prove the non-central moderate deviation result.

Proposition 4.3 (Non-central MD as $\mu \rightarrow \infty$ ). Assume that $\alpha \geq 1-\sqrt{\frac{1}{\beta}}$. Then, for every family of positive numbers $\left\{\varepsilon_{\mu}: \mu>0\right\}$ such that (6) holds, the family $\left\{\mu \varepsilon_{\mu} A_{x /\left(\mu \varepsilon_{\mu}\right)}(\beta \mu, \mu): \mu>0\right\}$ satisfies the $L D P$ with speed $1 / \varepsilon_{\mu}$, and good rate function $I_{2}$ (presented in Proposition 4.1).

Proof. We want to apply the Gärtner Ellis Theorem. So we have

$$
\begin{aligned}
\frac{1}{1 / \varepsilon_{\mu}} \log \mathbb{E}\left[e^{\frac{s}{\varepsilon_{\mu}} \mu \varepsilon_{\mu} A_{x /(\mu \varepsilon \mu)}(\beta \mu, \mu)}\right] & =\varepsilon_{\mu} \log \mathbb{E}\left[e^{\left.s \mu A_{x /(\mu \varepsilon \mu}\right)(\beta \mu, \mu)}\right] \\
= & \begin{cases}\varepsilon_{\mu} \log \frac{2 \alpha \beta \mu e^{x /(\mu \varepsilon \mu) \Lambda(s \mu ; \beta \mu, \mu)}}{2 \beta \mu(\alpha-1)+\beta \mu+\mu-2 s \mu+\sqrt{(\beta \mu+\mu-2 s \mu)^{2}-4 \beta \mu^{2}}} & \text { for } s \mu \leq \frac{(\sqrt{\beta \mu}-\sqrt{\mu})^{2}}{2} \\
\infty & \text { otherwise }\end{cases} \\
& = \begin{cases}\varepsilon_{\mu} \log \frac{2 \alpha \beta e^{x \Lambda(s ; \beta, 1) / \varepsilon}}{2 \beta(\alpha-1)+\beta+1-2 s+\sqrt{(\beta+1-2 s)^{2}-4 \beta}} & \text { for } s \leq \frac{(\sqrt{\beta}-1)^{2}}{2} \\
\infty & \text { otherwise; }\end{cases}
\end{aligned}
$$

then

$$
\left.\lim _{\mu \rightarrow \infty} \frac{1}{1 / \varepsilon_{\mu}} \log \mathbb{E}\left[e^{\frac{s}{\varepsilon_{\mu}} \mu \varepsilon_{\mu} A_{x /\left(\mu \varepsilon_{\mu}\right)}(\beta \mu, \mu)}\right]=x \Lambda(s ; \beta, 1) \text { (for all } s \in \mathbb{R}\right),
$$

and, by Remark 2.2, the desired LDP is a straightforward application of the Gärtner Ellis Theorem.

In the next remark we follow the same lines of Remarks 3.2 and 4.1 .

Remark 4.3 (A version of Proposition 4.3 with exposed points). Here we discuss what happens when we consider the inequality $\alpha<1-\sqrt{\frac{1}{\beta}}$ in Proposition 4.3 . In this case we still have to consider some items of in the second part of Lemma 2.1 (as in Remarks 3.2 and 4.1) and, by the Gärtner Ellis Theorem, we have

$$
\limsup _{\mu \rightarrow \infty} \frac{1}{1 / \varepsilon_{\mu}} \log P\left(\mu \varepsilon_{\mu} A_{x /\left(\mu \varepsilon_{\mu}\right)}(\beta \mu, \mu) \in F\right) \leq-\inf _{z \in F} x \Lambda^{*}(z / x ; \beta, 1, \alpha) \text { for all closed sets } F
$$

and

$$
\liminf _{\mu \rightarrow \infty} \frac{1}{1 / \varepsilon_{\mu}} \log P\left(\mu \varepsilon_{\mu} A_{x /\left(\mu \varepsilon_{\mu}\right)}(\beta \mu, \mu) \in G\right) \geq-\inf _{z \in G \cap \mathcal{E}} x \Lambda^{*}(z / x ; \beta, 1, \alpha) \text { for all open sets } G
$$

where $\mathcal{E}=(x \tilde{z}(\beta, 1, \alpha), \infty)$ is the set of exposed points of $x \Lambda^{*}(\cdot / x ; \beta, 1, \alpha)$. Note that $x \tilde{z}(\beta, 1, \alpha)>$ $z_{2}$, and therefore both $x \Lambda^{*}(\cdot / x ; \beta, 1, \alpha)$ and $I_{2}$ uniquely vanish at $z_{2}$.

We conclude with the analogue of Remark 4.2 , where we also give some comments on the limit of the scaled variance.

Remark 4.4 (The analogue of Remark 4.2). The class of LDPs in Proposition 4.3 fills the gap between the two following asymptotic regimes as $\mu \rightarrow \infty$ : 
- the convergence of $A_{x}(\beta \mu, \mu)$ to $x \Lambda^{\prime}(0 ; \beta, 1)$ (case $\left.\varepsilon_{\mu}=1 / \mu\right)$, which follows from the LDP in Proposition 4.1;

- the weak convergence of $\mu A_{x / \mu}(\beta \mu, \mu)$ to $A_{x}(\beta, 1)$ (case $\varepsilon_{\mu}=1$ ) proved in Lemma 4.1.

In both cases one condition in (6) holds, and the other one fails. We can also provide the following limit for the scaled variance (where we take into account the variance expression in (4)):

$$
\lim _{\mu \rightarrow \infty} \frac{1}{\varepsilon_{\mu}} \operatorname{Var}\left[\mu \varepsilon_{\mu} A_{x /\left(\mu \varepsilon_{\mu}\right)}(\beta \mu, \mu)\right]=x \Lambda^{\prime \prime}(0 ; \beta, 1) .
$$

Thus the variance of the equally distributed random variables in Lemma 4.1 (and therefore the variance of the weak limit $\left.A_{x}(\beta, 1)\right)$ can be expressed as

$$
\operatorname{Var}\left[A_{x}(\beta, 1)\right]=\frac{4\left(\beta+2 \beta x \alpha^{2}+2 \alpha-1\right)}{(\beta-1)^{3} \alpha^{2}}=\Delta(\alpha, \beta)+x \Lambda^{\prime \prime}(0 ; \beta, 1),
$$

where $x \Lambda^{\prime \prime}(0 ; \beta, 1)$ is the limit value obtained above, and $\Delta(\alpha, \beta):=\frac{4(\beta+2 \alpha-1)}{(\beta-1)^{3} \alpha^{2}}>0 ;$ moreover $\Delta(\alpha, \beta)$ tends to zero as $\beta \rightarrow \infty$.

\section{$5 \quad$ Numerical estimates by simulations}

In this section we refer to the asymptotic Normality result under the scaling 2 stated in Remark 4.2. We present numerical values obtained by simulations to estimate $\beta$; actually we assume that $\beta>\beta_{0}$ for some known $\beta_{0}>1$. In the final part we also present some figures concerning sample paths for some $\beta>1$.

We denote the standard Normal distribution by $\Phi$. Then, for every $\delta>0$, we have

$$
\lim _{\mu \rightarrow \infty} P\left(A_{x}(\beta \mu, \mu)-\frac{\delta}{\sqrt{\mu}} \leq \mathbb{E}\left[A_{x}(\beta \mu, \mu)\right] \leq A_{x}(\beta \mu, \mu)+\frac{\delta}{\sqrt{\mu}}\right)=2 \Phi\left(\frac{\delta}{\sqrt{8 \beta x /(\beta-1)^{3}}}\right)-1
$$

so, if we choose $\delta=\sqrt{\frac{8 \beta x}{(\beta-1)^{3}}} \Phi^{-1}\left(\frac{1+\ell}{2}\right)$ for some $\ell \in(0,1)$, the above limit is equal to $\ell$. Thus we can consider the following approximated confidence interval for $\mathbb{E}\left[A_{x}(\beta \mu, \mu)\right]$ at the level $\ell$, when $\mu$ is large:

$$
A_{x}(\beta \mu, \mu) \pm \sup _{\beta>\beta_{0}} \sqrt{\frac{8 \beta x}{(\beta-1)^{3}}} \frac{\Phi^{-1}\left(\frac{1+\ell}{2}\right)}{\sqrt{\mu}} .
$$

We already remarked just after Proposition 4.1 that

$$
\lim _{\mu \rightarrow \infty} \mathbb{E}\left[A_{x}(\beta \mu, \mu)\right]=x \Lambda^{\prime}(0 ; \beta, 1)=x \frac{\beta+1}{\beta-1} ;
$$

thus, for $\mu$ large enough $\left(\mu>\mu_{0}\right.$, say) the approximation $\mathbb{E}\left[A_{x}(\beta \mu, \mu)\right] \approx x \frac{\beta+1}{\beta-1}$ can be adopted. Moreover, since $\sup _{\beta>\beta_{0}} \sqrt{\frac{8 \beta x}{(\beta-1)^{3}}}=\sqrt{\frac{8 \beta_{0} x}{\left(\beta_{0}-1\right)^{3}}}$, the above approximated confidence interval can be specified as follows:

$$
A_{x}(\beta \mu, \mu) \pm \sqrt{\frac{8 \beta_{0} x}{\left(\beta_{0}-1\right)^{3}}} \frac{\Phi^{-1}\left(\frac{1+\ell}{2}\right)}{\sqrt{\mu}} .
$$

Then we can obtain numerical values for this confidence interval by performing simulations of $A_{x}(\beta \mu, \mu)$. Specifically, the validate simulations of $A_{x}(\beta \mu, \mu)$ are those performed for selected 
values of $\beta$, i.e. for chosen values $\beta=\beta_{*}>\beta_{0}>1$, for which the fraction of sample paths such that

$$
x \frac{\beta_{*}+1}{\beta_{*}-1} \in\left(\bar{A}_{x}\left(\beta_{*} \mu, \mu\right)-\sqrt{\frac{8 \beta_{0} x}{\left(\beta_{0}-1\right)^{3}}} \frac{\Phi^{-1}\left(\frac{1+\ell}{2}\right)}{\sqrt{\mu}}, \bar{A}_{x}\left(\beta_{*} \mu, \mu\right)+\sqrt{\frac{8 \beta_{0} x}{\left(\beta_{0}-1\right)^{3}}} \frac{\Phi^{-1}\left(\frac{1+\ell}{2}\right)}{\sqrt{\mu}}\right),
$$

where $\bar{A}_{x}\left(\beta_{*} \mu, \mu\right)$ is the simulated sample mean, is at least $\ell$; this is also equivalent to say that

$$
x \frac{\beta_{*}+1}{\beta_{*}-1}-\sqrt{\frac{8 \beta_{0} x}{\left(\beta_{0}-1\right)^{3}}} \frac{\Phi^{-1}\left(\frac{1+\ell}{2}\right)}{\sqrt{\mu}}<\bar{A}_{x}\left(\beta_{*} \mu, \mu\right)<x \frac{\beta_{*}+1}{\beta_{*}-1}+\sqrt{\frac{8 \beta_{0} x}{\left(\beta_{0}-1\right)^{3}}} \frac{\Phi^{-1}\left(\frac{1+\ell}{2}\right)}{\sqrt{\mu}} .
$$

Thus, when $\mu$ is large, $\beta$ can be estimated by the following items.

- The confidence interval at the level $\ell$, when $x<\bar{A}_{x}\left(\beta_{*} \mu, \mu\right)-\sqrt{\frac{8 \beta_{0} x}{\left(\beta_{0}-1\right)^{3}}} \frac{\Phi^{-1}\left(\frac{1+\ell}{2}\right)}{\sqrt{\mu}}$ :

$$
\left(\frac{\bar{A}_{x}\left(\beta_{*} \mu, \mu\right)+\sqrt{\frac{8 \beta_{0} x}{\left(\beta_{0}-1\right)^{3}}} \frac{\Phi^{-1}\left(\frac{1+\ell}{2}\right)}{\sqrt{\mu}}+x}{\bar{A}_{x}\left(\beta_{*} \mu, \mu\right)+\sqrt{\frac{8 \beta_{0} x}{\left(\beta_{0}-1\right)^{3}}} \frac{\Phi^{-1}\left(\frac{1+\ell}{2}\right)}{\sqrt{\mu}}-x}, \frac{\bar{A}_{x}\left(\beta_{*} \mu, \mu\right)-\sqrt{\frac{8 \beta_{0} x}{\left(\beta_{0}-1\right)^{3}}} \frac{\Phi^{-1}\left(\frac{1+\ell}{2}\right)}{\sqrt{\mu}}+x}{\bar{A}_{x}\left(\beta_{*} \mu, \mu\right)-\sqrt{\frac{8 \beta_{0} x}{\left(\beta_{0}-1\right)^{3}}} \frac{\Phi^{-1}\left(\frac{1+\ell}{2}\right)}{\sqrt{\mu}}-x}\right) .
$$

- The point estimation:

$$
\frac{\bar{A}_{x}\left(\beta_{*} \mu, \mu\right)+x}{\bar{A}_{x}\left(\beta_{*} \mu, \mu\right)-x}
$$

Moreover, in addition to these estimators, the meaningful information carried by these simulations concern both $\mu_{0}$ and $\beta_{*}$ for which the inequality (7) is satisfied.

Now we are ready to present some numerical values. In all cases we perform simulations by setting $x=1$ and $\beta_{0}=1.25$; furthermore, the size of simulated sample paths is $10^{3}$ and the confidence level is $\ell=0.95$.

Table 1: Numerical approximations for the confidence interval for $\beta$ varying $\alpha$

\begin{tabular}{||c||c|c|c|c|c|c}
\hline$\alpha$ & $\mu$ & $\beta_{*}$ & $x \frac{\beta_{*}+1}{\beta_{*}-1}$ & $\bar{A}_{x}\left(\beta_{*} \mu, \mu\right)$ & confidence interval (8) & point estimation (9) \\
\hline 0.7 & 1000 & 1.75 & $3 . \overline{6}$ & 5.15575 & $(1.349423,1.772864)$ & 1.481261 \\
\hline 0.8 & 1000 & 1.75 & $3 . \overline{6}$ & 4.496909 & $(1.394876,2.03684)$ & 1.571934 \\
\hline 0.9 & 1000 & 1.75 & $3 . \overline{6}$ & 4.03037 & $(1.434939,2.367616)$ & 1.659985 \\
\hline 0.925 & 1000 & 1.75 & $3 . \overline{6}$ & 3.92666 & $(1.444975,2.472007)$ & 1.683373 \\
\hline
\end{tabular}

Table 2: Numerical approximations for the confidence interval for $\beta$ varying $\mu$

\begin{tabular}{c||c||c|c|c|c|c}
\hline$\alpha$ & $\mu$ & $\beta_{*}$ & $x \frac{\beta_{*}+1}{\beta_{*}-1}$ & $\bar{A}_{x}\left(\beta_{*} \mu, \mu\right)$ & confidence interval (8) & point estimation $[9]$ \\
\hline 0.9 & 1000 & 2 & 3 & 3.297049 & $(1.517462,3.743193)$ & 1.870682 \\
\hline 0.9 & 5000 & 2 & 3 & 3.292031 & $(1.66817,2.257219)$ & 1.872588 \\
\hline 0.9 & 10000 & 2 & 3 & 3.292868 & $(1.717179,2.112946)$ & 1.87227 \\
\hline 0.9 & 20000 & 2 & 3 & 3.291491 & $(1.756974,2.030459)$ & 1.872794 \\
\hline
\end{tabular}

We conclude with some comments, and we also refer to the figures presented below. In Table 1. we have increasing values of $\alpha$ and we find decreasing values of the sample mean $\bar{A}_{x}\left(\beta_{*} \mu, \mu\right)$ (as expected) that tend to the asymptotic value $x \frac{\beta_{*}+1}{\beta_{*}-1}$. Furthermore, we find also increasing values of 
Table 3: Numerical approximations for the confidence interval for $\beta$ varying $\beta_{*}$

\begin{tabular}{c|c||c||c|c|c|c}
\hline$\alpha$ & $\mu$ & $\beta_{*}$ & $x \frac{\beta_{*}+1}{\beta_{*}-1}$ & $\bar{A}_{x}\left(\beta_{*} \mu, \mu\right)$ & confidence interval $(8)$ & point estimation $(9)$ \\
\hline 0.8 & 1000 & 1.5 & 5 & 6.128791 & $(1.298652,1.561668)$ & 1.389955 \\
\hline 0.8 & 1000 & 2 & 3 & 3.678367 & $(1.470994,2.80116)$ & 1.746724 \\
\hline 0.8 & 1000 & 2.5 & $2 . \overline{3}$ & 2.860925 & $(1.583278,7.827018)$ & 2.074734 \\
\hline 0.8 & 1000 & 2.75 & $2 . \overline{142857}$ & 2.626983 & $(1.625987,34.89173)$ & 2.229269 \\
\hline
\end{tabular}

the point estimate $(9)$ and wider confidence intervals. In Table 2 , for increasing values of $\mu$, we find quite stable values for $\bar{A}_{x}\left(\beta_{*} \mu, \mu\right)$ and the point estimation $(9)$. Moreover we obtain more and more narrow confidence intervals as $\mu$ increases. In Table 3 , it appears evident that, for increasing values of $\beta_{*}$, the values of the sample mean $\bar{A}_{x}\left(\beta_{*} \mu, \mu\right)$ become more accurate estimations for the corresponding values of $x \frac{\beta_{*}+1}{\beta_{*}-1}$, whereas the right endpoints of the confidence intervals are less accurate. We also remark that, in all tables, the estimated values based on the point estimation (9) are less than the corresponding set values of $\beta_{*}$.

From Tables 1 3 , and all performed simulations results, we can say that the numerical strategy to obtain the above estimates is reliable for high values of $\alpha$. This is easily understandable because the above estimates are reliable in a neighborhood of the asymptotic value $x \frac{\beta_{*}+1}{\beta_{*}-1}$ (for high value of $\mu$, i.e. for high rate of downward steps) or, in some sense equivalently, for high value of absorbing probability $\alpha$ (compare Figure 1 and left side of Figure 2). Furthermore, as far as the value $\mu_{0}$ is concerned (i.e. the value such that we can obtain reliable estimates, at the confidence level 0.95, when $\mu>\mu_{0}$ ), we can take $\mu_{0}=1000$. Furthermore, the results in Table 2 show that the approximation of the confidence interval improves as $\mu$ increases. Finally we also stress that all these numerical values provide indications on the true value of $\beta$ under the scaling 2 for finite values of $\mu$ (instead of asymptotic results as $\mu \rightarrow \infty$ ).

We conclude with some brief comments on Figures 1,2. They show that sample paths of the process for different choices of values for parameters $\mu, \alpha, \beta$. In Figure 1 it is possible to observe how the paths change for different values of $\mu$. In Figure 2 we consider different values of $\alpha$ and $\beta$; in particular, we set $\mu=10$ because the effect of different values of $\alpha$ and $\beta$ on the sample paths appears more evident.

\section{References}

[1] Crimaldi I, Di Crescenzo A, Iuliano A, Martinucci B (2013) A generalized telegraph process with velocity driven by random trials. Adv Appl Prob 45:1111-1136

[2] Dembo A, Zeitouni O (1998) Large Deviations Techniques and Applications, 2nd edn Springer, New York

[3] Di Crescenzo A, Martinucci B (2010) A damped telegraph random process with logistic stationary distribution. J Appl Prob 47:8496

[4] Di Crescenzo A, Zacks S (2015) Probability law and flow function of Brownian motion driven by a generalized telegraph process. Methodol Comput Appl Probab 17:761-780

[5] Di Crescenzo A, Martinucci B, Zacks S (2018) Telegraph process with elastic boundary at the origin. Methodol. Comput. Appl. Probab. 20:333-352

[6] De Gregorio A, Orsingher E (2011) Flying randomly in $\mathbb{R}^{d}$ with Dirichlet displacements. Stoch Proc Appl 122:676-713 




Figure 1: Sample paths for different values of $\mu$, for $\alpha=0.9$ and $\beta=1.25$.
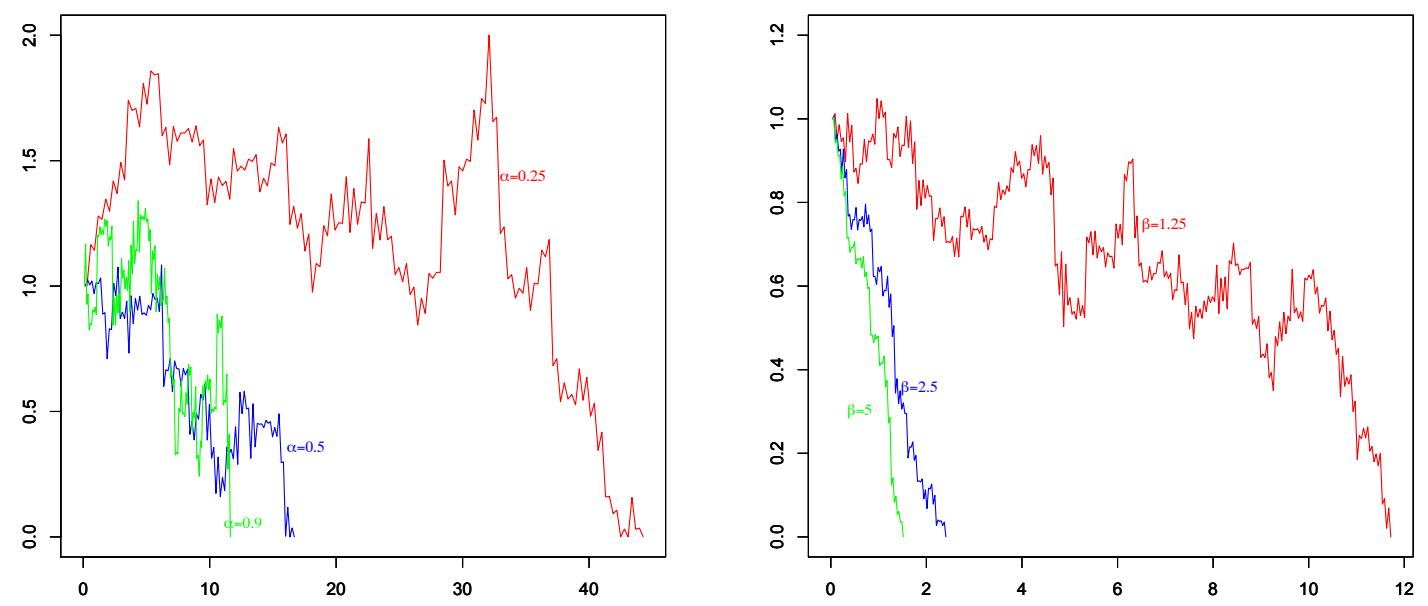

Figure 2: Left: sample paths for different values of $\alpha$, for $\mu=10$ and $\beta=1.25$. Right: the same varying $\beta$, for $\mu=20$ and $\alpha=0.9$. 
[7] Dominé M (1995) Moments of the first-passage time of a Wiener process with drift between two elastic barriers. J Appl Prob 32:1007-1013

[8] Dominé M (1996) First passage time distribution of a Wiener process with drift concerning two elastic barriers. J Appl Prob 33:164-175

[9] Foong SK (1992) First-passage time, maximum displacement, and Kac's solution of the telegrapher equation. Phys Rev A 46:R707-R710

[10] Garra R, Orsingher E (2014) Random flights governed by Klein-Gordon-type partial differential equations. Stoch Proc Appl 124:2171-2187

[11] Giorno V, Nobile AG, Pirozzi E, Ricciardi LM (2006) On the construction of first-passage-time densities for diffusion processes. Sci Math Jpn 64:277-298

[12] Goldstein S (1951) On diffusion by discontinuous movements, and on the telegraph equation. Quart J Mech Appl Math 4:129-156

[13] Jacob E (2012) A Langevin process reflected at a partially elastic boundary: I. Stoch Proc Appl 122:191-216

[14] Jacob E (2013) Langevin process reflected on a partially elastic boundary II. Séminaire de Probabilités XLV:245-275, Lecture Notes in Math, 2078, Springer, Cham

[15] Kac M (1974) A stochastic model related to the telegrapher's equation. Rocky Mountain J Math 4:497-509

[16] Macci C (2011) Large deviation results for wave governed random motions driven by semiMarkov processes. Comm Statist Simulation Comput 40:1342-1363.

[17] Macci C (2016) Large deviations for some non-standard telegraph processes. Statist Probab Lett 110:119-127.

[18] Orsingher E (1990) Probability law, flow function, maximum distribution of wave-governed random motions and their connections with Kirchoff's laws. Stoch Proc Appl 34:49-66

[19] Orsingher E (1995) Motions with reflecting and absorbing barriers driven by the telegraph equation. Random Oper Stochastic Equations 3:9-21

[20] Ratanov NE (1997) Random walks in an inhomogeneous one-dimensional medium with reflecting and absorbing barriers. Theoret Math Phys 112:857-865

[21] Ratanov N (2015) Telegraph processes with random jumps and complete market models. Methodol Comput Appl Probab 17:677-695

[22] Stadje W, Zacks S (2004) Telegraph processes with random velocities. J Appl Prob 41:665-678 